\title{
DETALLES ULTRAESTRUCTURALES DE LA MADERA DE ALGUNAS BORAGINACEAE DE MÉXICO
}

\author{
JOSEFINA BARAJAS MORALES**
}

\section{RESUMEN}

Se investigó la presencia de puntuaciones ornamentadas y de inclusiones de diversos tipos por medio del microscopio electrónico de barrido en cinco especies de Cordia y una de Bourreria.

Las cinco especies de Cordia presentaron cristales de oxalato de calcio y tílides mientras que en $B$. purpusii se encontraron cristales pero no tílides. En $C$. alliodora, C. elaeagnoides, $C$. dentata y $B$. purpussi fueron encontradas puntuaciones ornamentadas; en C. alliodora y $C$. dentata se localizó almidón en las fibras y el parénquima.

\begin{abstract}
Vestured pits and different kinds of inclusions was searched for under the scanning microscope in five species of Cordia and one of Bourreria.

Calcium oxalate crystals and tyloses were found in all five species of Cordia while in $B$. purpusii crystals were found but not tyloses.

Vestured pits were found in $C$. alliodora, $C$. elaeagnoides and $B$. purpussi, starch was also found in the fibres and parenchyma of $C$. alliodora y $C$. dentata.
\end{abstract}

\section{INTRODUCGIÓN}

La importancia taxonómica de las puntuaciones ornamentadas en las familias que las presentan es bien conocida (Bailey 1933); no obstante, en 1977 Miller efectuó un estudio sobre la presencia de ornamentación en las puntuaciones de la familia Boraginaceae, encontrándola presente sólo en algunas especies de los géneros Echium, Pteleo-

*Recibido para publicación en octubre de 1983.

**Instituto de Biología, Universidad Nacional Autónoma de México. Ap. Postal 70-233, 04510 México, D.F.

Barajas-Morales J. 1983. Detalles utraestructurales de la madera de algunas Boraginaceae de México. Boletín de la Sociedad Botánica de México 45: 3-14. 
carpa y Rochefortia, por lo que dedujo que en esta familia la presencia de ornamentaciones no tiene implicación taxonómica.

Amos (1952) en su revisión de las familias botánicas con presencia de sílice en el parénquima no menciona a las Boraginaceae; no obstante Gottwald en 1980 sugirió que la presencia de diversas inclusiones, tales como el sílice y los cristales de oxalato de calcio, tienen valor diagnóstico para esta familia, por lo que se hace necesario conocer bien el tipo de inclusiones presentes en cada uno de los taxa que la integran.

Asimismo, en el trabajo previo sobre anatomía de las Boraginaceae (Barajas-Morales, 1981), también se hizo patente la necesidad de conocer la ultraestructura de las puntuaciones para detectar la ornamentación y además para confirmar los tipos de inclu-siones presentes en cada especie.

\section{MATERIALES Y MÉTODOS}

Fueron estudiadas seis especies de Boraginaceae provenientes de 12 árboles colectados cerca de la Estación de Biología de Chamela, situada en el km 59 de la carretera Barra de Navidad-Puerto Vallarta, en el estado de Jalisco. Las especies estudiadas y sus números de registro en la xiloteca del Instituto de Biología, MEXU son: Cordia alliodora (Ruiz et Pav.) Cham., 130, 166; C. dentata Poir., 165, 178; C. elaeagnoides D.C., 149, 168; C. hintonii Johnst., 160, 171; C. sonorae Rose, 131, 167; Bourreria purpusii Brandeg., 170 y 179 .

Las muestras consistieron en bloques de $5 \mathrm{~mm}$ de lado obtenidos aproximadamente a $1.50 \mathrm{~m}$ de altura en el tronco principal; en cada bloque se obtuvo una superficie lisa tangencial o radial usando una navaja nueva para cada corte (Exley, Meylan y Butterfield, 1977); estas muestras fueron cubiertas con carbón y oro y analizadas en un microscopio electrónico de barrido JEOL JSM-35.

\section{RESULTADOS}

Todas las especies estudiadas presentaron cristales prismáticos de oxalato de calcio en el parénquima axial y radial (Figs. 3, 7, 11, 15, 19, 23 y 24). Todas las especies

\section{Figuras 1 a 4 . Cordia alliodora}

Fig. 1. Puntuaciones intervasculares no ornamentadas $4000 \mathrm{X}$.

Fig. 2. Puntuación intervascular ornamentada $10000 \mathrm{X}$.

Fig. 3. Cristales de oxalato de calcio en parénquima de rayo $860 \mathrm{X}$.

Fig. 4. Granos de almidón en el lumen de una fibra $2400 \mathrm{X}$.

\section{Figuras 5 a 8 . Cordia dentata}

Fig. 5. Puntuaciones intervasculares lisas y ornamentadas mostrando un retículo que circunda la cámara $4000 \mathrm{X}$.

Fig. 6. Puntuación intervascular ornamentada mostrando un retículo que circunda la cámara $8600 \mathrm{X}$.

Fig. 7. Cristales de oxalato de calcio y almidón (A) en parénquima radial $1000 \mathrm{X}$.

Fig. 8. Grano de almidón en el parénquima axial $3000 \mathrm{X}$. 
ULTRAESTRUCTURA DE LA MADERA DE BORAGINACEAE
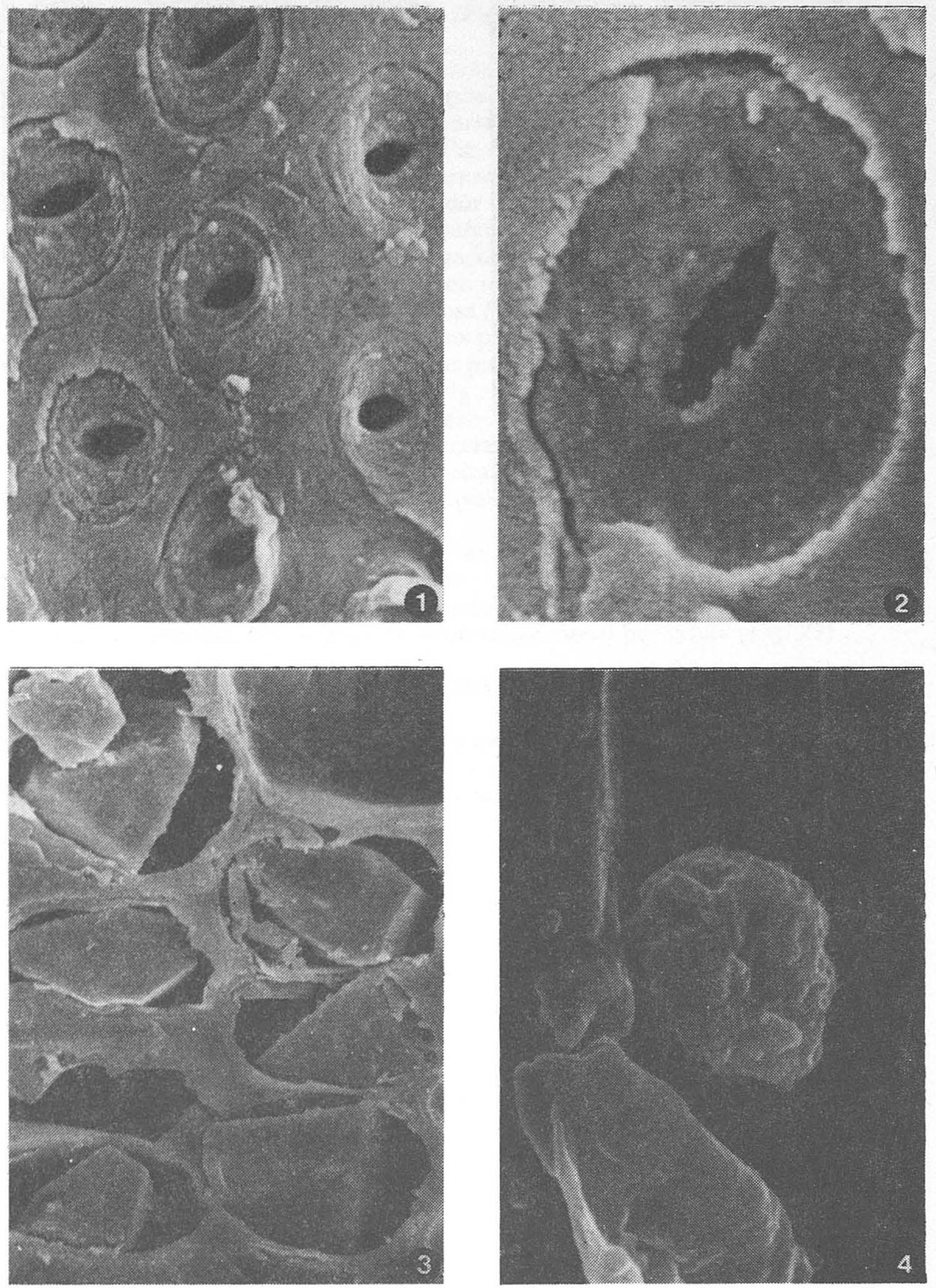
BOLETÍN DE LA SOCIEDAD BOTÁNICA DE MÉXICO No. 45, 1983
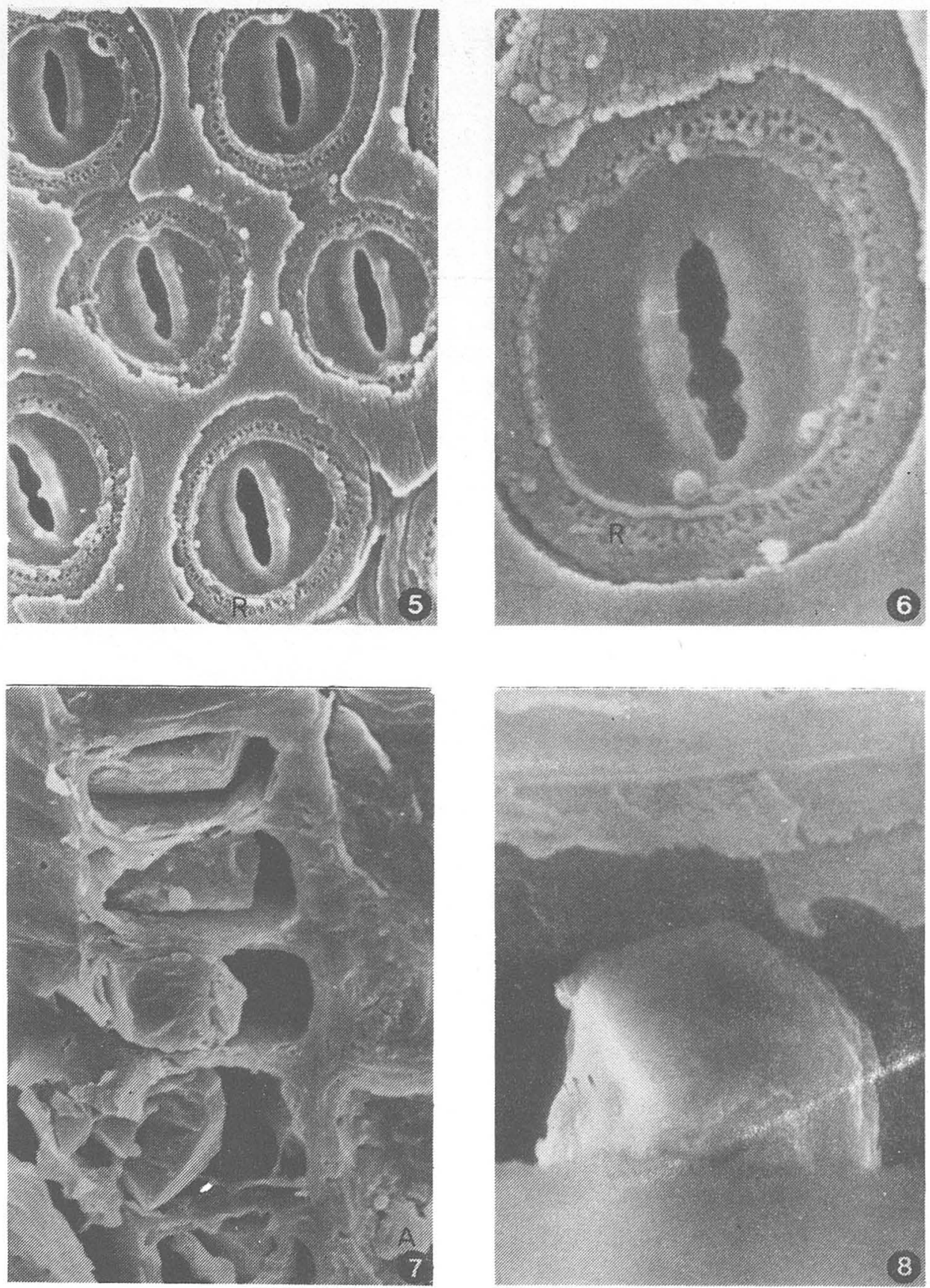


\section{ULTRAESTRUCTURA DE LA MADERA DE BORAGINACEAE}

de Cordia presentaron tílides en los vasos (Fig. 26) pero éstas no estuvieron presentes en Bourreria.

C. alliodora tiene puntuaciones no ornamentadas (Fig. 1) y otras con pequeñas proyecciones hacia su abertura, las cuales a gran aumento aparecen como ornamentaciones (Fig. 2). Se confirmó además la presencia de granos de almidón en el parénquima axial y radial (Fig. 25), fibras y vasos (Fig. 4).

En C. dentata fueron observadas puntuaciones claramente ornamentadas (Figs. 5 y 6), que presentaron un retículo alrededor de la cámara (R), además de granos de almidón en las fibras (Fig. 28) y en el paŕenquima axial y radial (Fig. 8)

El punteado radiovascular fue alargado horizontalmente (Fig. 7).

Las puntuaciones de C. elaeagnoides son ornamentadas (Figs. 9 y 10 ), densamente cubiertas por material de la capa verrucosa (v) o no ornamentadas. En el interior de los vasos se observaron también depósitos probablemente de sílice (Fig. 12).

De $C$. hintonii pudieron ser apreciadas puntuaciones íntervasculares sin areola, no ornamentadas (Fig. 13) y coalescentes (Fig. 14). También fue observada en el lumen de una fibra, una inclusión que por su aspecto y tamaño parece ser sílice (Fig. 16).

Para $C$. sonorae se comprobó la presencia de sílice en el parénquima radial y axial (Fig. 20) y se observaron las puntuaciones intervasculares no ornamentadas y sin areola (Figs. 17 y 18) en las que se aprecian claramente las vesículas de la llamada capa verrucosa (v).

Bourreria purpusii presenta puntuaciones intervasculares muy pequeñas $(3 \mathrm{u})$ pero claramente ornamentadas (Figs. 21 y 22).

En el parénquima radial y axial se presentan varios cristales de muy diferentes tamaños en una sola célula (Fig. 23) o un cristal único por célula (Fig. 24).

\section{DISCUSIÓN}

En $B$. purpusii se presentaron puntuaciones ornamentadas bien definidas. En $C$. alliodora y $C$. dentata se observaron sólo como pequeñas proyecciones hacia la abertura, y en $C$. elaeagnoides se notaron muy recubiertas por material de la capa verrucosa, no

Figuras 9 a 12. Cordia elaeagnoides

Fig. 9. Puntuaciones intervasculares ornamentadas $4800 \mathrm{X}$.

Fig. 10. Puntuación intervascular ornamentada $10000 \mathrm{X}$.

Fig. 11. Cristales de oxalato de calcio en el parénquima radial $1000 \mathrm{X}$.

Fig. 12. Pequeños granulos probablemente de sílice adheridos a la pared interna de un vaso $1600 \mathrm{X}$.

\section{Figuras 13 a 16 . Cordia hintonii}

Fig. 13. Puntuaciones intervasculares no ornamentadas y sin areola $2200 \mathrm{X}$.

Fig. 14. Puntuaciones intervasculares coalescentes $800 \mathrm{X}$.

Fig. 15. Cristales de oxalato de calcio en parénquimà radial $600 \mathrm{X}$.

Fig. 16. Grumo probablemente de sílice en el lumen de una fibra $1600 \mathrm{X}$. 
BOLETÍN DE LA SOCIEDAD BOTÁNICA DE MÉXICO No. 45, 1983
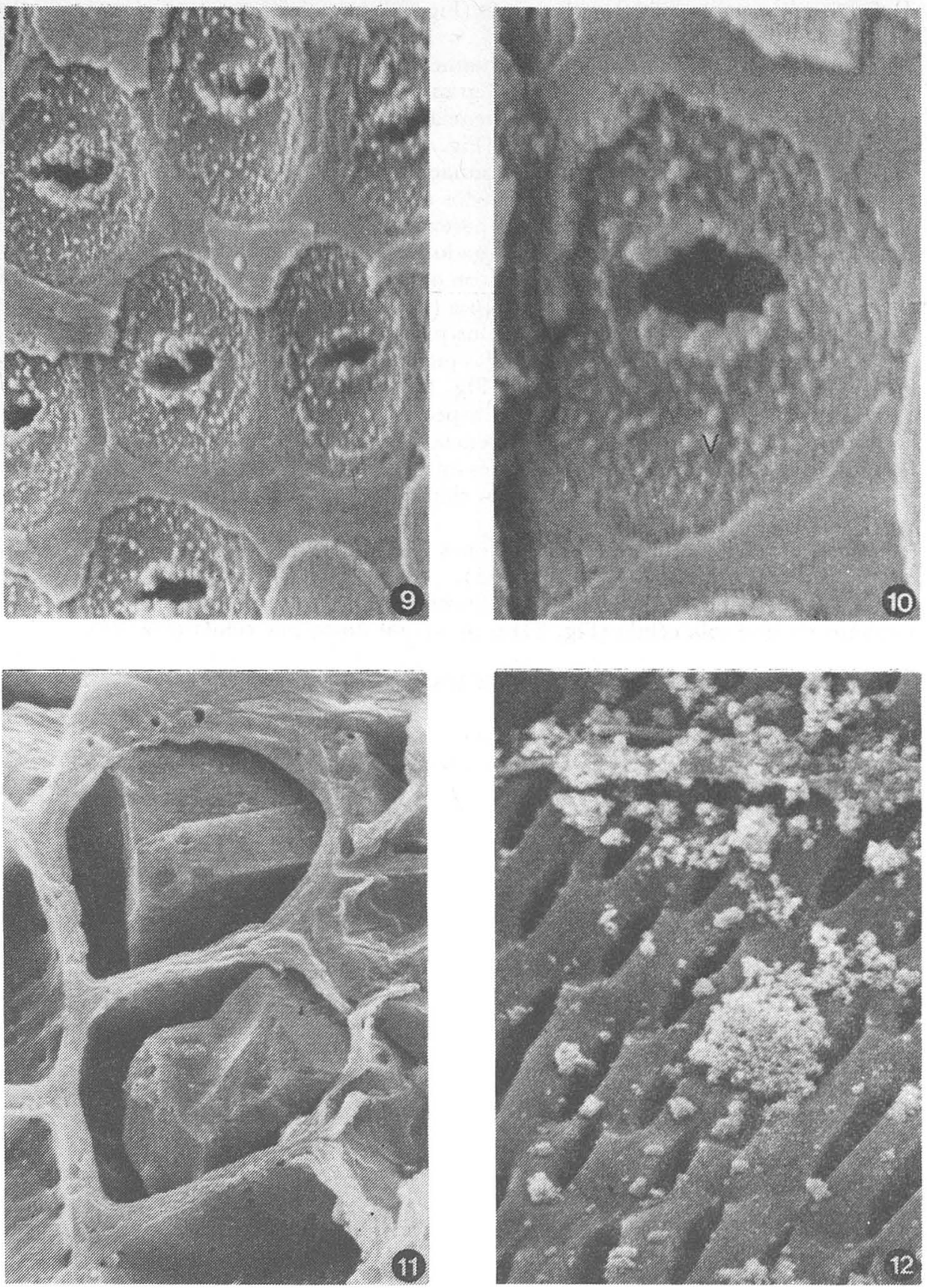
ULTRAESTRUCTURA DE LA MADERA DE BORAGINACEAE
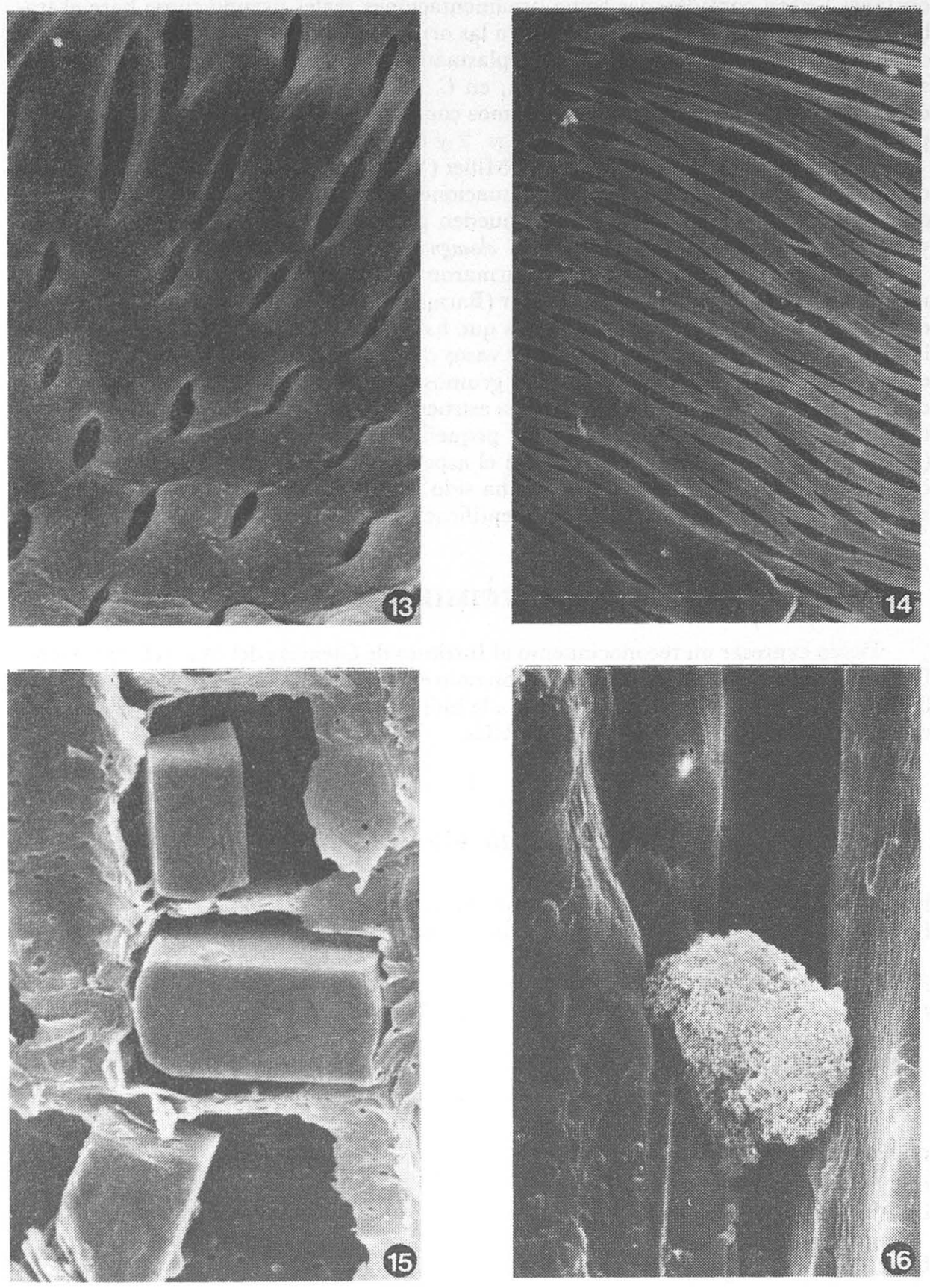
obstante fueron consideradas como ornamentaciones reales usando como base el trabajo de Schmid (1965) en el que define a las ornamentaciones como parte de la pared de la puntuación debido a su origen citoplasmático, a partir de vesículas que depositan su contenido no fibrilar sobre la areola; en $C$. alliodora y $C$. dentata se distingue muy claramente que las proyecciones que hemos considerado como ornamentación forman parte de la pared de la puntuación (Figs. 2 y 6).

Por otra parte apoyamos la idea de Miller (1977) sobre la carencia de validez taxonómica de la ornamentación en las puntuaciones de las Boraginaceae, pues incluso se observó que en el mismo ejemplar se pueden presentar puntuaciones ornamentadas y no ornamentadas como sucede en C. elaeagnoides y C. alliodora.

Con respecto a inclusiones se confirmaron en general los resultados obtenidos al microscopio óptico en el trabajo anterior (Barajas-Morales, 1981) pero también se encontraron algunos detalles estructurales que habían pasado desapercibidos como son los grumos de sílice en el interior de los vasos de $C$. elaeagnoides los granos de almidón en las fibras de $C$. dentata y los pequeños grumos, probablemente de sílice, en las fibras de C. hintonii; también fue observada una estructura que circunda la cámara de la puntuación y que está integrada por áreas pequeñas sin depósito de material celulósico (Figs. 5 y 6 ) que en conjunto presentan el aspecto de un retículo (R) alrededor de la cámara, esta es una estructura que no ha sido citada en la literatura y por ahora no sabemos cuál puede ser su valor en identificación o taxonomía.

\section{AGRADECIMIENTOS}

Deseo expresar mi reconocimiento al Instituto de Ciencias del Mar y Limnología, UNAM, por permitirme el uso del microscopio electrónico de barrido, para el desarrollo de este trabajo y muy especialmente a la bióloga Yolanda Hornelas, encargada del uso del aparato, quien tomó las fotografías.

\section{Figuras 17 a 20. Cordia sonorae}

Fig. 17. Puntuaciones intervasculares no ornamentadas y sin areola $2700 \mathrm{X}$.

Fig. 18. Puntuaciones intervasculares sin areola en las que es muy evidente la capa verrucosa (v) $5000 \mathrm{X}$.

Fig. 19. Cristales de oxalato de calcio en parénquima radial $1100 \mathrm{X}$.

Fig. 20. Grumo de sílice en parénquima radial 3600 X.

\section{Figuras 21 a 24 . Bourreria purpusii}

Fig. 21. Puntuaciones intervasculares ornamentadas $8600 \mathrm{X}$.

Fig. 22. Puntuaciones intervasculares ornamentadas $18000 \mathrm{X}$.

Fig. 23. Cristales de oxalato de calcio de diversos tamaños en el parénquima radial $1100 \mathrm{X}$.

Fig. 24. Cristal de oxalato de calcio, en célula de rayo $2200 \mathrm{X}$. 
ULTRAESTRUCTURA DE LA MADERA DE BORAGINACEAE
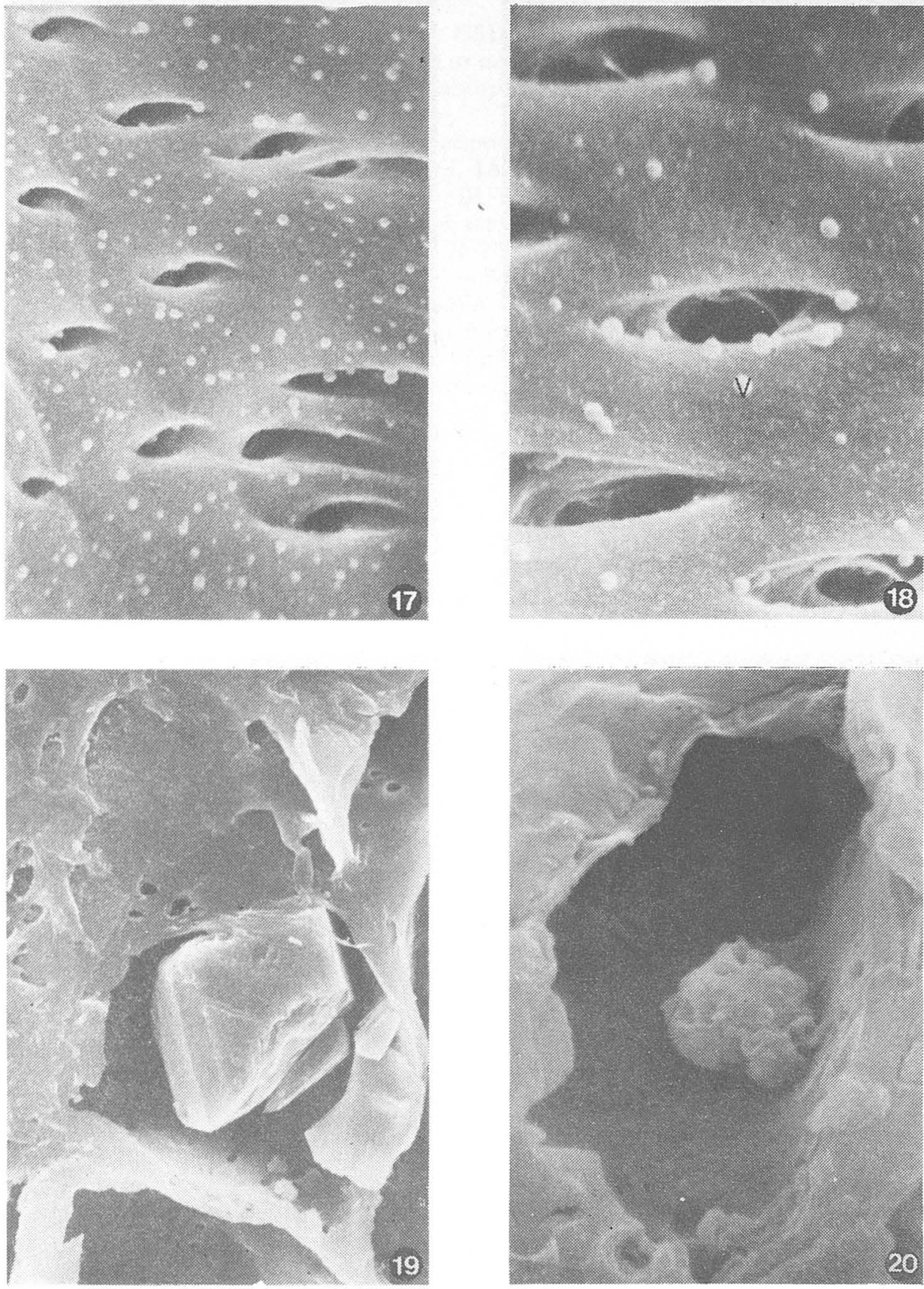
BOLETÍN DE LA SOCIEDAD BOTÁNICA DE MÉXICO No. 45, 1983
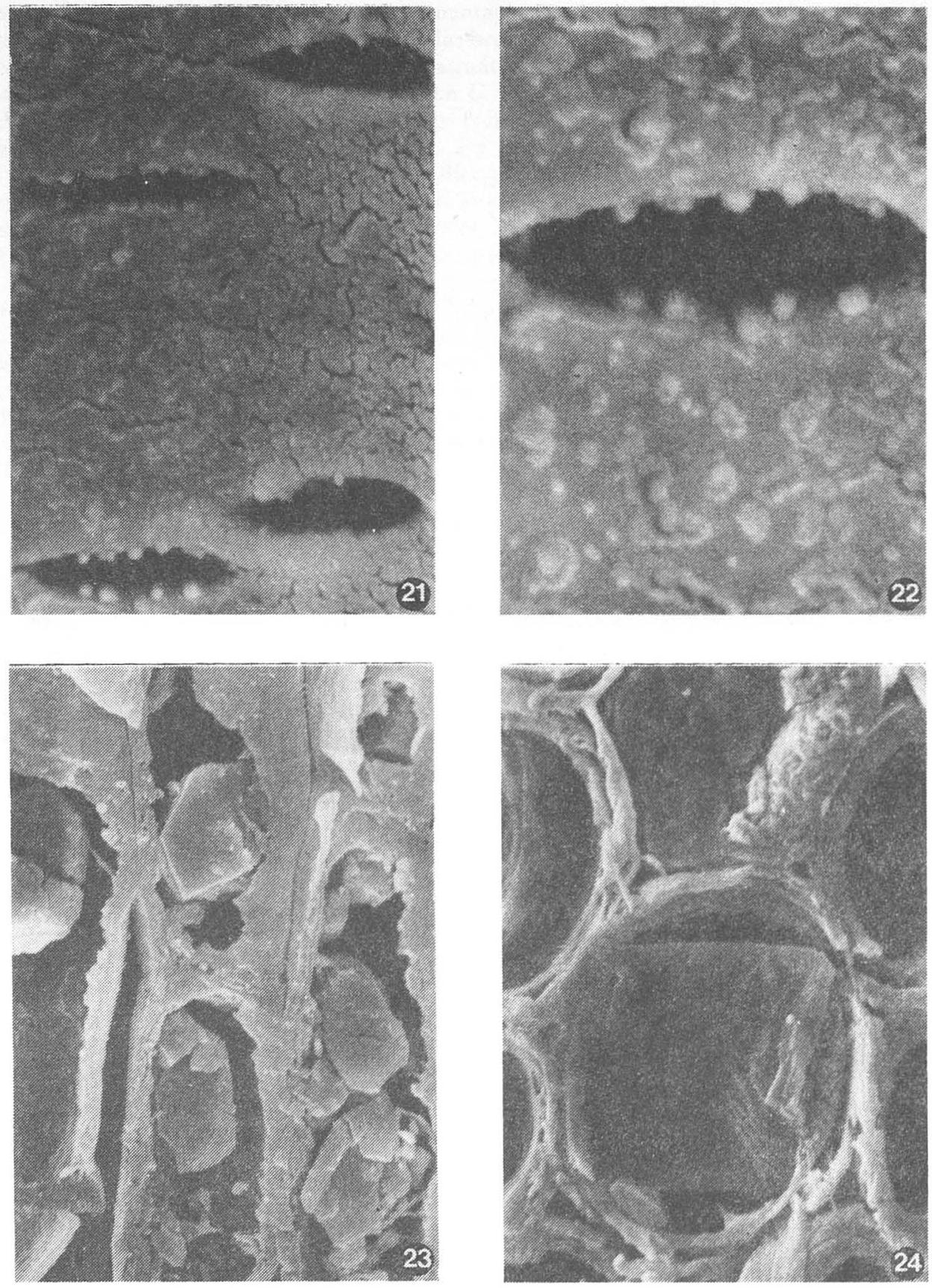


\section{BIBLIOGRAFÍA}

AMOS, G.L. 1952. Silica in Timbers. CSIRO Bull Melbourne.

BAILEY, I.W. 1933. The cambium and its derivative tissues. VIII. Structure, distribution and diagnostic significance of vestured pits in dicotyledons. J. Arnold Arbor. 14: 259-273.

BARAJAS-MORALES, J. 1981. Description and notes on the wood anatomy of Boraginaceae from western Mexico. IAWA Bull n.s. 2 (2-3): 61-67.

EXLEY, R.R., B.A. MEYLAN y B.G. BUTTERFIELD. 1977. A technique for obtaining clean cut surfaces on wood samples prepared for the scanning electron microscope. J. Microsc. 110 (1): 75-78.

GOTTWALD, H. 1980. 'Louro Preto' - Found to be the first silica bearing Cordia (Cordia glabrata, Boraginaceae). IAWA Bull n.s. 1 (1-2): 55-58.

MILLER, R.B. 1977. Vestured pits in Boraginaceae. IAWA Bull. 3: 43-48.

SCHMID, R. 1965. The fine structure of pits in hardwoods. pp. 291-304. En. W.A. Cote, Jr. (Ed.) Cellular ultrastructure of woody plants. Syracuse University Press. Syracuse, New York.

Figuras 25 a 28. Cordia spp.

Fig. 25. Granos de almidón (A) y cristales de oxalato de calcio en parénquima radial de C. alliodora $1100 \mathrm{X}$.

Fig. 26. Tílide en C. dentata $430 \mathrm{X}$.

Fig. 27. Puntuaciones radiovasculares alargadas horizontalmente en $C$. dentata.

Fig. 28. Grano de almidón el lumen de una fibra en $C$. dentata $2400 \mathrm{X}$. 
BOLETÍN DE LA SOCIEDAD BOTÁNICA DE MÉXICO No. 45, 1983
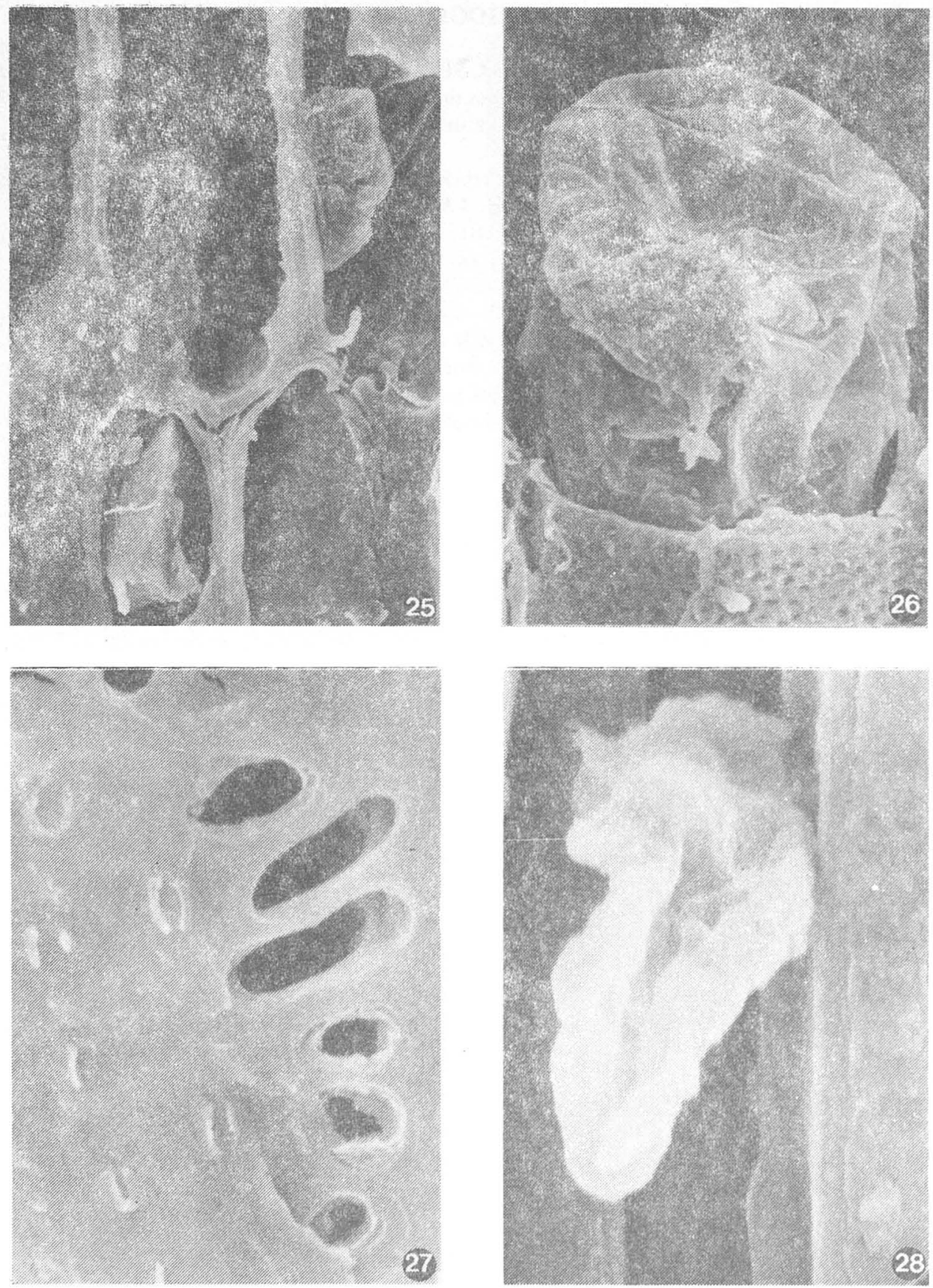